\title{
Congenital Variations Discovered in the Clinical Presentation of Hyperkeratosis of the Hand and Foot: A report of 2 cases
}

\author{
by Al Kline, $\mathrm{DPM}^{1} \square$
}

The Foot \& Ankle Journal 2 (1): 3

Case presentations describing a congenital variation of palmoplantar keratosis are presented. The majority of these conditions are autosomal dominant with associated nail dystrophy. A variant condition is described with little palmar keratosis; however, finger nail and toe nail dystrophy is the most common identifying feature. Gene identification and treatment protocol are presented. Fortunately, these conditions are rare. A good knowledge of these conditions will help in proper diagnosis and treatment.

Key words: Palmoplantar keratosis (PPK), hand, foot, congenital, hyperkeratosis

Accepted: December, 2008

Published: January, 2009

This is an Open Access article distributed under the terms of the Creative Commons Attribution License. It permits unrestricted use, distribution, and reproduction in any medium, provided the original work is properly cited. @The Foot \& Ankle Journal (www.faoj.org)

Congenital hyperkeratosis is an uncommon condition of the foot. There are a number of congenital conditions that cause hyperkeratotic lesions of the foot. Fortunately, these conditions remain rare in the population. These lesions can be divided into diffuse and punctuate. There is also a subclass of hereditary diseases defined as either epidermolytic or nonepidermolytic. Conditions characterized by palmoplantar keratosis (PPK) are the most causes of congenital hyperkeratosis. These conditions include UnnaThost disease, Vohwinkel's syndrome, PapillonLefèvre syndrome and pachyonychia congenita. ${ }^{1,2,3,4}$

Address correspondence to: $\mathrm{Al}$ Kline, DPM

3130 South Alameda, Corpus Christi, Texas 78404.

1 Adjunct Clinical Faculty, Barry University School of Podiatric Medicine. Private practice, Chief of Podiatry, Doctors Regional Medical Center. Corpus Christi, Texas, 78411.
Unna-Thost disease was first described in 1880 by Herrmann Arthur Thost and again described in 1883 by Paul Gerson Unna., ${ }^{5,6,7}$ Synonyms for the disease include Brünauer-Fuhs-Siemens syndrome, Brauer's syndrome and Brünauer's syndrome. ${ }^{7}$ It is a disease of autosomal dominant origin characterized by severe palmoplantar keratosis. Deep fissures and hypohidrosis with thickened skin of the palms of the hands and soles of the feet usually occur within the first year after birth. ${ }^{1}$ Vohwinkel's syndrome or keratoderma hereditaria mutilans is a rare autosomal dominant condition first described in 1929. ${ }^{2}$ Clinical manifestations first appear in infants and then proceed through childhood into adulthood. Keratosis described as honeycomb and starfish-like in appearance is common. In the later stages of the disease, pseudoainhum or auto amputation of the digits can occur due to constricting bands of keratosis around the digit. 


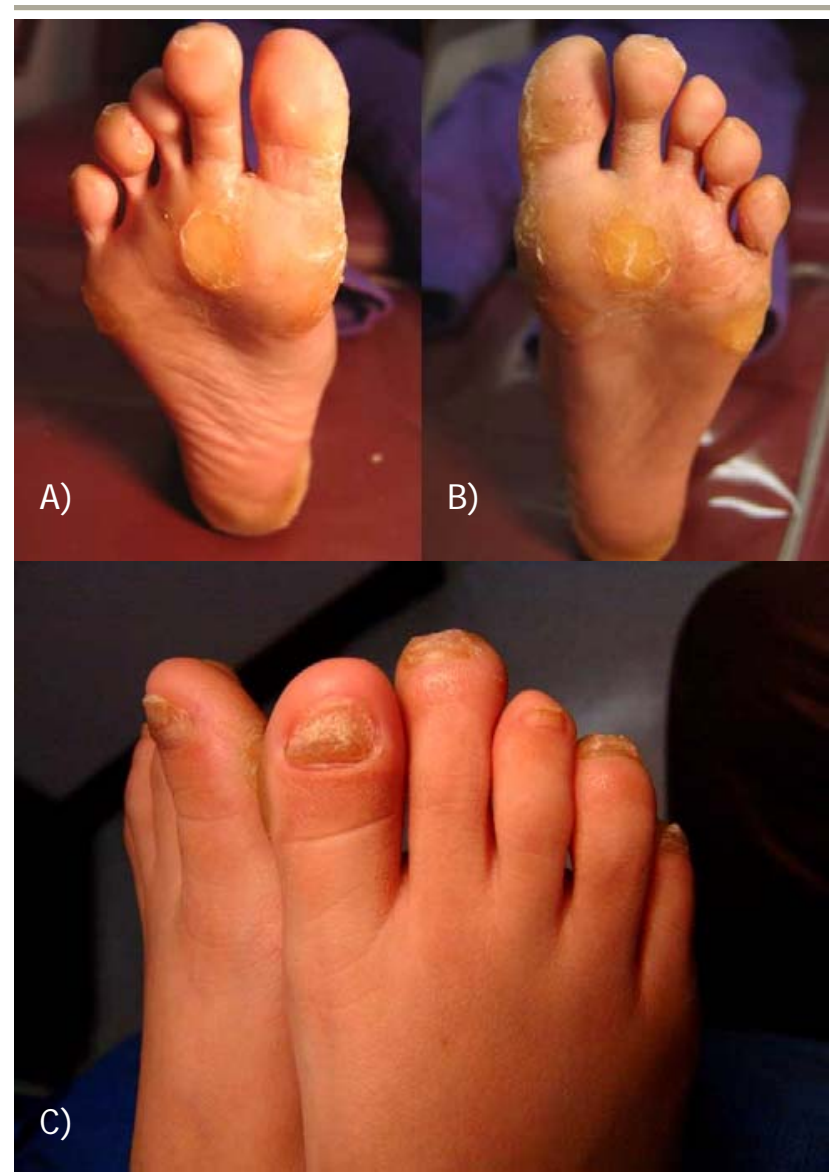

Figure 1 A 9 year old-female with severe plantar regions of hyperkeratosis ( $A$ and $B$ ). The toe nails are severely dystrophic $(C)$. This condition began in infancy and is congenital.

In fact, pseudoainhum is characteristic in a number of hereditary hyperkeratosis. ${ }^{1,2,3,4}$ PapillonLefèvre syndrome (PLS), also known as Mal de Meleda , was first described by two French physicians in $1924 .^{3,8,9}$ It is an extremely rare genodermatosis inherited as an autosomal recessive trait, affecting children between the ages of 1-4. ${ }^{3}$ Psoriatic-like plaques involving the palms, soles and elbows are described that worsen in winter and are often hyperhidrotic resulting in a foul odor. ${ }^{3}$ Pachyonychia congenita (PC) is a rare genodermatosis that affects the nails of all the toes and fingers. ${ }^{4}$ Other names and synonyms of this condition are called congenital dyskeratosis and pachyonychia ichthyosiformis.
Most cases appear within the first or second years of life, although cases of late onset have been reported in the second and third decades. (which is termed PC tarda) ${ }^{4}$ Diagnostic features include symmetrical thickening of skin, dysmorphic nails and hyperkeratotic skin lesions. The disease fits into two major types: Jadassohn-Lewandowsky syndrome (JLS-1) and Jackson-Lawler syndrome (JLS-2). It is an autosomal dominant trait. Some recessive forms have been described. JLS-1 is characterized by nail hypertrophy, nail dystrophy, PPK, follicular keratosis and oral leukokeratosis and is the most common form of PC $(56.2 \%){ }^{4}$ JLS-2 usually lacks oral leukokeratosis and is commonly associated with epidermolytic bullae of the palms and soles $(24.9 \%){ }^{4}$

Secondary symptoms are commonly associated with hereditary hyperkeratosis. This is called complex keratodermas. Unna-Thost disease is commonly associated with secondary fungal and bacterial infections. ${ }^{1}$ Corneal opacities, pilitorti, hearing loss, hypohidrosis and dental abnormalities have also been described. ${ }^{7}$

Vohwinkel's syndrome can be associated with deafness, cancer, cardiomyopathy and adrenal insufficiency. ${ }^{2}$ PLS is often associated with severe periodontitis which usually starts at the age of three or four. ${ }^{3}$ This is seen with gingivitis and rapid destruction of the periodontium when deciduous teeth proceed normally. Other associated conditions of PLS include pyogenic liver abscesses with impaired immunodeficiency. ${ }^{3}$ The most common secondary associated symptom with PC is oral leukokeratosis with associated periodontitis and loss of teeth. ${ }^{4}$ The teeth develop normally and are lost within 1 year.

\section{Gene Identification}

Often, the clinical presentation is not as straight forward as the texts present. Clinical evaluation can present with diffuse as well as punctuate keratodermas and associated nail dystrophy. Clinical diagnosis is usually made by presentation and secondary associated conditions in complex keratodermas. 


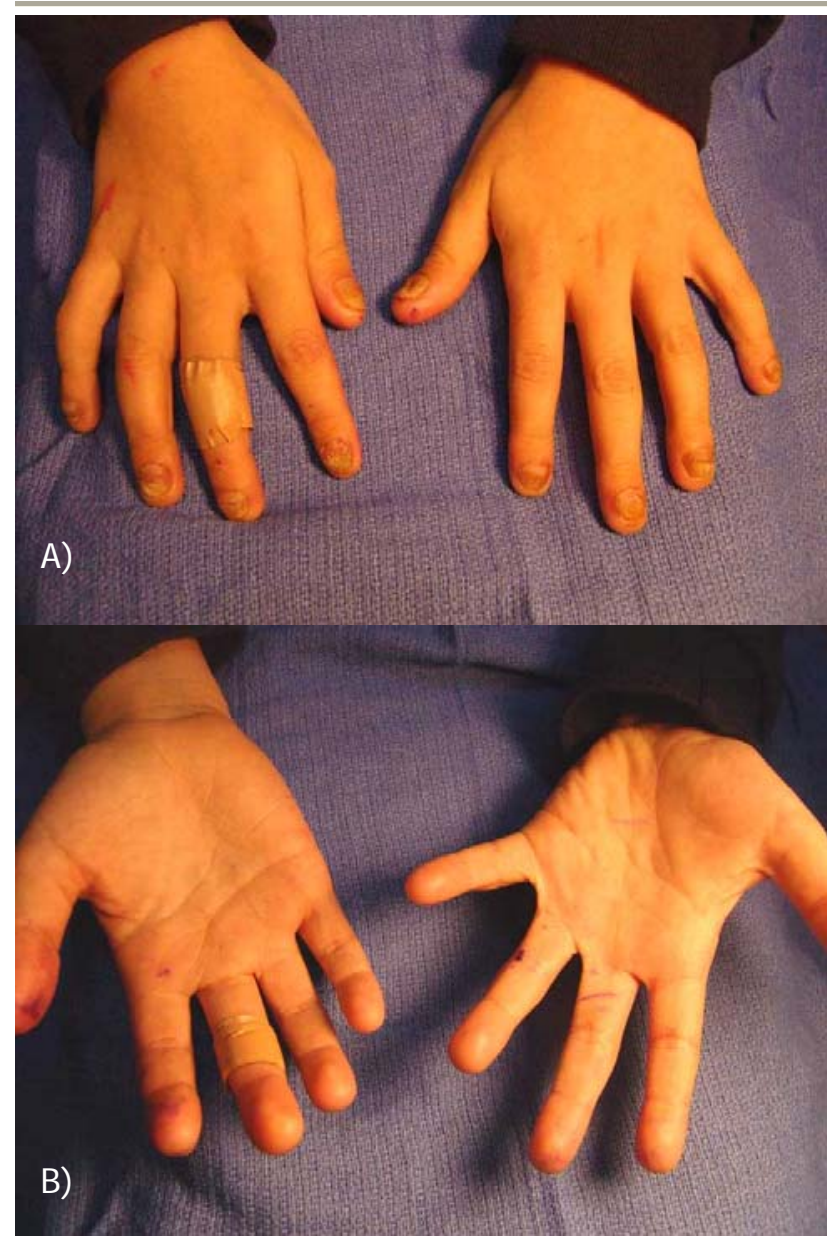

Figure 2 Although there is severe plantar congenital hyperkeratosis, the hands show very little palmar keratosis ( $A$ and $B$ ).

Recent molecular biological studies indicate the presence of two variants of Vohwinkel's syndrome, an ichthyosis-associated variant, associated with an insertional mutation of the loricrin gene, and a deafness-associated variant, associated with a missense mutation of the connexin-26 gene. ${ }^{2}$ In PLS, there is a reported loss-of-function mutation affecting both alleles of the cathepsin- $C$ gene identified on chromosome 11q14.1-q14.3. ${ }^{3}$ In Pachyonychia congenita (PC) mutations in the KRT16 and KRT 17 gene encoding keratins K6a and K16 (KRT16) that can trigger JLS-1 and JLS-2 respectively have recently been identified. ${ }^{4}$

\section{Case Studies}

Case 1: A 9 year-old female presents to our office with diffuse and punctate hyperkeratosis of both feet. (Fig. 1) Clinically, the hyperkeratosis is located on the soles of both feet with associated severe nail dystrophy to all fingers and toes. Interestingly, there is little palmar hyperkeratosis. (Fig. 2) The patient's father, grandmother and aunt are affected with the same condition. All have PPK with associated fingernail and toenail dystrophy and discoloration. The patient presented with the condition at birth. The patient's grandfather and uncle are asymptomatic. The condition is characterized by extreme pain.

The patient's father has been treated with narcotics for a number of years. Most of the adults in the family abuse tobacco. The father, grandmother and aunt have undergone multiple surgical debridements in attempts to reduce keratosis. This included surgical debridement of deep keratomas, removal of nail plates and beds and metatarsal head compression osteotomies and $5^{\text {th }}$ metatarsal head resections. To date, surgery was only temporarily effective.

Retinoids were not used at the time. The family did not exhibit any complex symptoms and dentition was normal. No oral leukokeratosis was seen on examination. The entire family has diffuse hyperkeratosis and hyperkeratosis of all nails affecting both hands and feet symmetrically.

The daughter is now treated routinely treated with Retinoid creams, keratolytic agents, debridement and accommodative padding on a regular basis. It appears that most family members affected had the condition worse on the soles of the feet than the hands. Although there is very little palmar keratosis in the 9 year-old female, both the nails of the hands and feet are affected. The patient's hands reveal discolored, dystrophic changes to the finger nails, but very little palmer keratosis. 


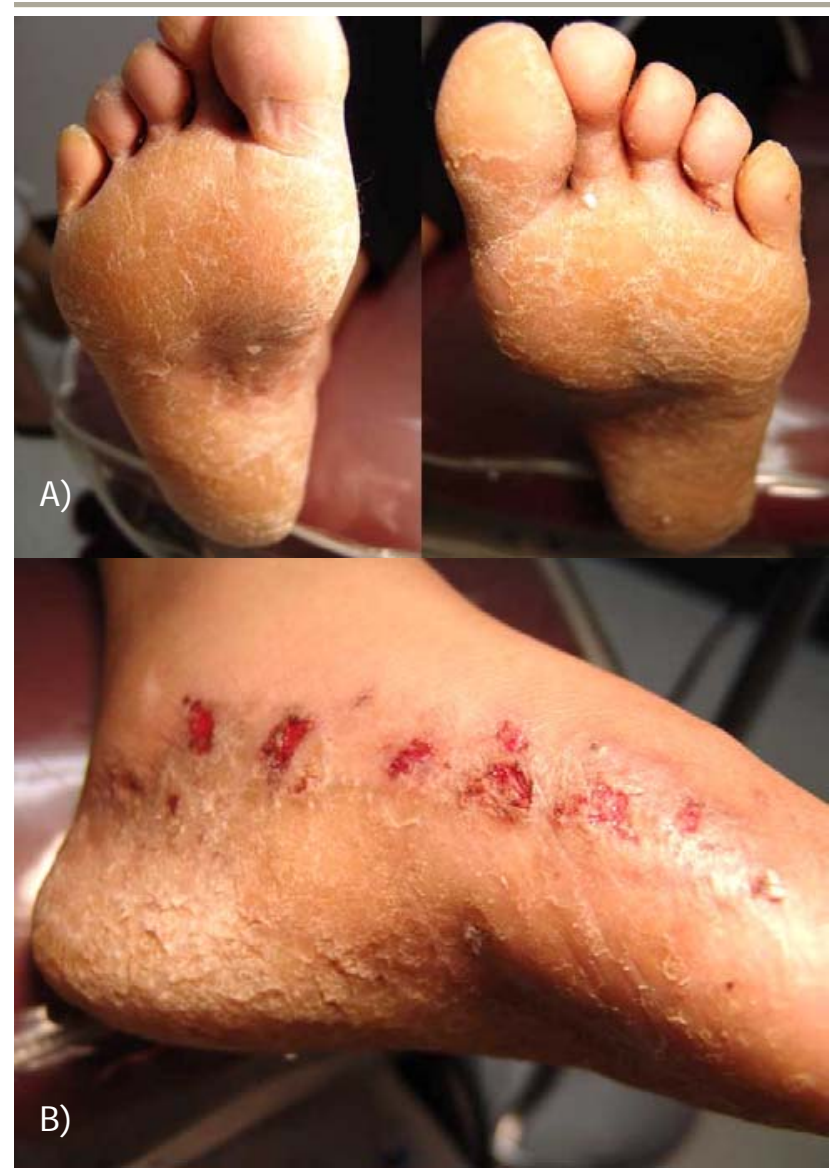

Figure 3 A 39 year-old female with diffuse keratosis to both feet $(A)$. Secondary infectious tinea is also observed along the medial border of the foot (B). In this variant form, the hyperkeratosis is more diffuse rather than punctate along the soles of the feet.

Case 2: A 37 year-old female presents with severe plantar keratoderma and secondary inflammation and interdigital bacterial infection. She has allergies to iodine and seafood. The patient has diffuse plantar foot keratosis with nail involvement. (Fig. 3) However, her hands appeared to be almost spared of the condition. There is some distal darkening just under the distal region of the finger nail. (Fig. 4).

Her family history shows that most family members were affected by the disease. Her grandmother had the condition, but not the grandfather. They had eight children, 4 boys and 4 girls.
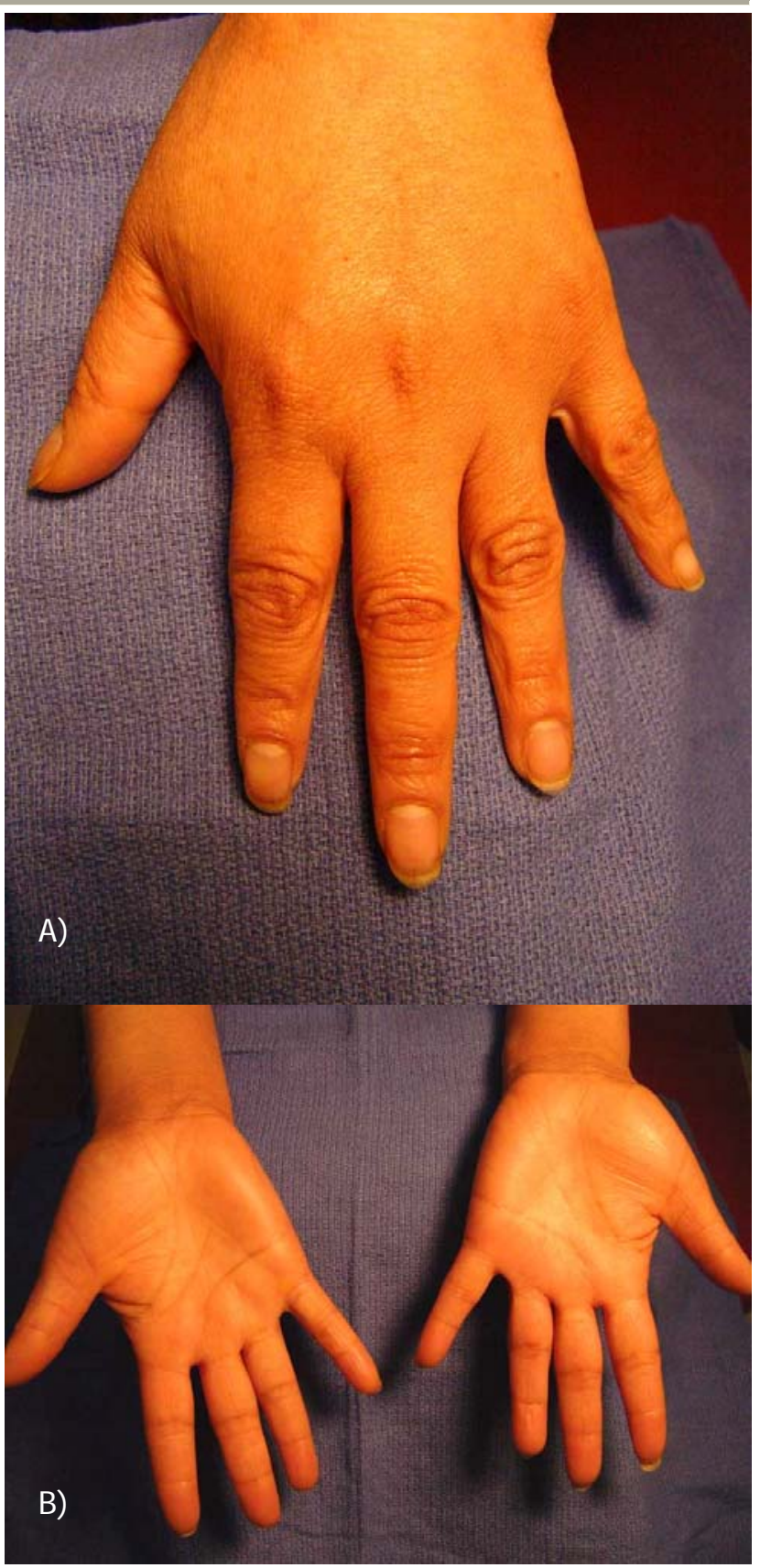

Figure 4 As in case 1, this 39 year-old female has very little palmar keratosis. Distal nail discoloration is observed ( $A$ and $B$ ).

All of the boys inherited the disease and only one girl. Three girls were unaffected. Her mother (who is unaffected) and father (who is one of the boys affected) had 2 boys and 4 girls. Of this group, her 2 oldest sisters have the condition and one brother is also affected. 
Only one male was unaffected. Some of the siblings have the condition much worse than the others.

\section{Treatment}

Treatment regimes are based on surgical techniques, medications and ancillary treatments designed to decrease painful keratosis. Surgical debridement or paring of keratosis is effective, but only temporary. Surgical bone debridement under regions of intense hyperkeratosis rarely works in our experience. There is very little information in the literature concerning full thickness surgical removal of tissue and skin grafting. Unfortunately, hyperkeratotic areas may return as soon as 1-2 weeks following simple debridement. Medications designed to improve PPK include keratolytic agents and oral or topical retinoids. The most common keratolytic agent used today is Vanamide $\AA$. Vanamide is a keratolytic, emollient cream designed to soften the skin. This can be used topically under occlusion for the best results. We have used Vanamide under occlusion for 3 or 4 days before the office visit. It helps most in the debridement of keratosis by softening or hydrating the region of hard keratosis. Vanamide's main ingredient is $40 \%$ urea, so it is especially useful in nail as well as skin debridement. ${ }^{10}$ Probably, the most widely used oral retinoid is Accutane or Isotretinoin. Retinoids are a class of medications derived from vitamin $A$ and used to treat various skin conditions from psoriasis to warts to skin cancers. Oral retinoids were first released for use in the United States and Europe in 1982. ${ }^{11}$ Oral accutane is most commonly used for cystic acne. Etretinate and Acitretin are more commonly used in the treatment of hyperkeratosis. ${ }^{11}$ Oral retinoids should be carefully used in females. The drugs are teratogenic causing serious birth defects. ${ }^{11}$ However, when carefully used, they have been found to be very effective in the treatment of various PPK disorders. ${ }^{2}$ There have also been reports of elevated liver enzymes while taking oral retinoids, so careful monitoring of the liver enzyme panel is recommended. ${ }^{11}$
Topical and ancillary treatments can include saline soaks, topical Vaseline under occlusion, adding bleach to bath water, antibacterial soaps and a host of others too numerous to mention.

\section{Discussion}

Hereditary hyperkeratotic disorders appear to be heterogenous in nature. In the case studies presented, the autosomal dominant gene has no predilection for males or females and is randomized, passing the trait to some siblings while sparing others. Autosomal dominant carriers have a 50:50 chance of passing this gene on to their siblings and the individuals spared will not have the ability to pass on this disease and will not be carriers. ${ }^{12}$ It also appears that genetic polymorphisms and mutations continue to occur in varied cases. This would explain why some individuals have the condition worse and some have milder forms of the disease. It can be safe to say that the majority of PPK disorders can have variant forms and severity. As varied as this disease can present, treatment results can also vary. We have found that aggressive debridement with use of topical keratolytics with oral retinoids provide some of the best results. Surgical procedures should only address regions that are most problematic and don't respond readily to conservative treatment regimes. Educational instruction and understanding should include a thorough discussion with your patients that results can vary and may be unsuccessful or only temporary. Discussing the disease and treatment options will enable better care of the patient with this frustrating condition. 


\section{References}

1. Kline A. Keratotic lesions of the foot. Podiatry Internet Journal 1 (1): 8, 2006.

2. Bari AU. Keratoderma hereditarium mutilans (Vohwinkel syndrome) in three siblings. Dermatology Online Journal. 12 (7): 10, 2006.

3. Janjua SA, Khachemoune A. Papillon-Lefèvre syndreom: Case report and review of the literature. Dermatology Online Journal. 10 (1): 13, 2004.

4. Caproni M, Fabbri P. Pachyonychia congenitalOrphanet Encylopedia., (online PDF) accessed 21/12/2008.

5. Thost A. Über erbliche Ichtyosis palmaris et plantaris cornea. Dissertation. Heidelberg, 1880.

6. Unna PG. Über das Keratoma palmare et plantare hereditarium. Vierteljahrsschrift für Dermatologie und Syphilis. Wien. 15: 231, 1883.

7. Unna Thost Syndrome. Who named it? (online), accessed 21/12/2008.

8. Papillon MM, Lefèvre P. Deux cas de kératodermie palmaire et plantaire symétrique familiale (maladie de Meleda) chez le frère et la soeur. Coexistence dans les deux cas d'altérations dentaires graves.

Bulletin de la Société française de dermatologie et de vénéorologie. 4 (31): 82-87, 1924.

9. Papillon MM. Lefèvre syndrome. Who named it? (online), accessd 22/12/2008.

10. Vanamide Cream (online PDF) , accessed $21^{\text {st }}$

December 2008.

11. Chan A, Hanna M, Abbott M, Keane R. Oral retinoids and pregnancy. The Medical Journal of Australia. 165: 164167, 1996.

12. The Universe of Genetic Testing, online resource. [online], accessed date 21/12/2008. 